\title{
Preoperative Levosimendan in heart failure patients undergoing hip fracture repair: a preliminary report
}

\author{
Martín Lorenzo M.D.C., González Fariña V., Montón Giménez N., Brito Do Carmo M.A., Torres Dios J.A., Solera \\ Marín J., Hospital Universitario de Canarias, Dept. of Anaesthesiology, La Laguna, Spain
}

\section{Introduction}

Elder patients with heart failure undergoing noncardiac surgery suffer substantial cardiac morbidity and mortality.

Levosimendan is a promising adjunct in our therapeutic repertoire for the treatment of cardiac failure. It's a novel calcium sensitizer, enhances myocardial contractility while simultaneously has vasodilatory and cardioprotective properties. This could be advantageous in perioperative management of heart failure patients. Our Objective was to evaluate the safety and efficiency of prophylactic preoperative levosimendan administration in these patients.

\section{Methods}

We studied 12 patients with heart failure and left ventricular ejection fraction $<45 \%$ undergoing hip fracture repair. Levosimendan was administrated with an infusion rate of 0.1 $\mu \mathrm{g} / \mathrm{kg} / \mathrm{min}$ in a total dose of $12,5 \mathrm{mg} 24$ hours prior to surgery. Hemodynamic and tissue perfusion parameters were obtained at baseline, 24 hours, 48 hours and 7 days after initiation of levosimendan.

\section{Results}

Twelve patients ( 7 females and 5 males) were studied. The average age of the patients was 86 with a 7 year derivations, and the average ejection fraction (EF) was 29 ( $\pm 5 \%)$. Patient's characteristics are given in Table 1.

TABLA 1 Characteristics demographics and preoperative risk stratification of the patients

\begin{tabular}{|c|c|c|c|c|c|c|c|}
\hline Patient & Age & Gender & LVEF & ASA & NYHA & LEE & Charlson \\
\hline 1 & 84 & M & 25,7 & III & III & II & 7 \\
\hline 2 & 92 & F & 32 & III & - & II & 5 \\
\hline 3 & 81 & F & 34,4 & III & - & III & 7 \\
\hline 4 & 92 & F & 43 & III & III & II & 2 \\
\hline 5 & 98 & F & 45 & III & II & I & 4 \\
\hline 6 & 87 & F & 40 & III & II & I & 1 \\
\hline 7 & 80 & F & 30 & III & - & II & 5 \\
\hline 9 & 67 & M & 32,8 & IV & III & IV & 7 \\
\hline 10 & 92 & F & 34,7 & III & - & II & 1 \\
\hline 12 & 93 & M & 33 & III & III & IV & 10 \\
\hline
\end{tabular}

ASA: American Society of Anesthesiologists Classification; Charlson: Comorbidity Index; LEE: Revised Cardiac Index by Lee; LVEF: Left Ventricular Ejection Fraction; NYHA: New York Heart Association.

The administration of Levosimendan caused a significant increment of:

- Left ventricular ejection fraction (LVEF) from $35 \pm 6$ to 43 $\pm 15 \%$ after 7 days (p 0,05$)$;

* Aortic velocity time integral (VTI) from $16 \pm 6 \mathrm{~cm}$ at baseline to $18 \pm 7 \mathrm{~cm}$ after 7 days (p 0,03 );

* Cardiac index from $3 \pm 0.6 \mathrm{1} / \mathrm{min} / \mathrm{m}^{2}$ at baseline to $3.6 \pm 0.7$ $1 / \mathrm{min} / \mathrm{m}^{2}$ after $24 \mathrm{~h}$ and $3,7 \pm 0.8$ after $48 \mathrm{~h}$ of an increased stroke volume index (baseline $35 \pm 11 \mathrm{ml} / \mathrm{m} 2$, after $24 \mathrm{~h} 42 \pm 9$ $\mathrm{ml} / \mathrm{m} 2$, after $48 \mathrm{~h} 43 \pm 8 \mathrm{ml} / \mathrm{m}^{2}(\mathrm{P}<0.02)$.

* Oxygen saturation in venous blood from $60 \pm 7 \%$ at baseline to $68 \pm 8 \%$ after 48 hours (P 0,01$)$.
The administration of Levosimendan caused a significant decrease:

- NT-proBNP plasma concentrations from $9253 \pm 11864 \mathrm{pg} / \mathrm{L}$ at baseline to $7961 \pm 10808 \mathrm{pg} / \mathrm{L}$ after $48 \mathrm{~h}(\mathrm{P} 0.04)$.

- Average arterial pressure from $86 \pm 14$ to $77 \pm 13 \mathrm{mmHg}$ in $48 \mathrm{~h}$

The administration of norepinephrine was necessary in a dose of $0.05-0.3 \mu \mathrm{g} / \mathrm{kg} / \mathrm{min}$ to prevent hypotension in 4 patients.

Hemodynamic changes exerted by levosimendan persisted up to $48 \mathrm{~h}$ (P 0,016).

The course of patient's haemodynamics is presented in Table 2. Severe adverse effects of levosimendan were not observed during the study.

TABLA 2 Effects of levosimendan on haemodynamics

\begin{tabular}{|c|c|c|c|c|c|c|}
\hline & Baseline & 24 hours & 48 hours & 7 days & $\begin{array}{c}P \\
\text { (baseline vs } \\
48 \text { hours) }\end{array}$ & $\begin{array}{c}P \\
\text { (baseline } \\
\text { vs 7days) }\end{array}$ \\
\hline LVEF (\%) & $35 \pm 6$ & $40 \pm 14$ & $41 \pm 16$ & $43 \pm 15$ & 0,2 & 0,05 \\
\hline VTI $(\mathrm{cm})$ & $16 \pm 6$ & $18 \pm 6$ & $19 \pm 7$ & $18 \pm 7$ & 0,02 & 0,03 \\
\hline $\mathrm{Cl}(1 / \mathrm{min} / \mathrm{m} 2)$ & $3 \pm 0,6$ & $3,6 \pm 0,7$ & $3,7 \pm 0,8$ & - & 0,02 & - \\
\hline $\mathrm{SVI}(\mathrm{ml} / \mathrm{m} 2)$ & $35 \pm 11$ & $42 \pm 9$ & $43 \pm 8$ & - & 0,02 & - \\
\hline $\mathrm{MAP}(\mathrm{mmHg})$ & $86 \pm 13,6$ & $77 \pm 13$ & $77 \pm 13$ & - & 0,02 & - \\
\hline HR (bpm) & $84 \pm 13$ & $85 \pm 14$ & $85 \pm 16$ & - & 0,7 & - \\
\hline CVP $(\mathrm{mmHg})$ & $11 \pm 8$ & $6 \pm 3$ & $8 \pm 2$ & - & 0,15 & - \\
\hline $\begin{array}{c}\mathrm{DO} 2 \\
\left(\mathrm{ml} / \mathrm{min} / \mathrm{m}^{2}\right)\end{array}$ & $\begin{array}{c}745 \pm \\
169\end{array}$ & $\begin{array}{c}820 \pm \\
158\end{array}$ & $710 \pm 242$ & - & 0,36 & - \\
\hline $\mathrm{SvO} 2(\%)$ & $60 \pm 6,9$ & $65,5 \pm 11$ & $67,6 \pm 8,1$ & - & 0,01 & - \\
\hline $\begin{array}{c}\text { NT-ProBNP } \\
(\mathrm{pg} / \mathrm{L})\end{array}$ & $\begin{array}{l}9253 \pm \\
11864\end{array}$ & $\begin{array}{c}6740 \pm \\
9530\end{array}$ & $\begin{array}{c}7961 \pm \\
10808\end{array}$ & $\begin{array}{c}8096 \pm \\
9107\end{array}$ & 0,04 & 0,9 \\
\hline
\end{tabular}

$\mathrm{Cl}$ : cardiac index; CVP: central venous pressure; DO2: oxygen delivery; HR: heart rate; LVEF: Left ventricular ejection fraction; MAP: mean arterial pressure; NTproBNP: N-terminal pro-B-type natriuretic peptide, SVI: stroke volumen index SvO2: oxygen saturation in venous blood; $\mathrm{VTI}$ : aortic velocity time integral

\section{Conclusion}

In patients with heart failure, preoperative initiation of levosimedan infusion improve periorepative haemodynamic parameters. These findings suggest that levosimendan could be an inotropic of great utility in preoperative optimization of cardiac function in high-risk patients undergoing major surgery due to its mechanism of action and its substained hemodynamic after having finished its infusion. 